\title{
A definition of the Ponzano-Regge quantum gravity model in terms of surfaces
}

\author{
Junichi Iwasaki \\ Physics Department, University of Pittsburgh, Pittsburgh, PA 15260, USA \\ E-mail: iwasaki@phyast.pitt.edu \\ April 14, 1995; revised on May 8, 1995
}

\begin{abstract}
We show that the partition function of the Ponzano-Regge quantum gravity model can be written as a sum over surfaces in a $(2+1)$ dimensional space-time. We suggest a geometrical meaning, in terms of surfaces, for the (regulated) divergences that appear in the partition function.
\end{abstract}

This paper is dedicated to the 5,100 people killed by the Kobe earthquake in Japan on January 17, 1995.

\section{Introduction}

The Ponzano-Regge quantum gravity model has been a mystery since the time of its construction [回]. Ponzano and Regge found an unexpected relation between the $6 \mathrm{j}$-symbol and Regge's discrete version of the action functional of (Euclidean) general relativity in 3-dimensions. The description of the relation follows. Consider a 3-dimensional simplex manifold, which consists of tetrahedra whose faces are attached to one another. Pick a tetrahedron and denote the lengths of its edges by $l_{s}(s=1,2, \cdots, 6)$ such that $\left(l_{1}, l_{2}, l_{3}\right)$, $\left(l_{3}, l_{4}, l_{5}\right),\left(l_{1}, l_{5}, l_{6}\right)$ and $\left(l_{2}, l_{6}, l_{4}\right)$ form the four faces of the tetrahedron. (See Fig. 1.) The Regge action for the tetrahedron is

$$
S_{\text {Regge }}=\sum_{s=1}^{6} l_{s} \theta_{s}
$$

where $\theta_{s}$ is the angle between the outward normals of two faces sharing the $s$-th edge. If we assign non-negative half-integer values $j_{s}(s=1,2, \cdots, 6)$ to the edges such that $l_{s}=\sqrt{j_{s}\left(j_{s}+1\right)}\left(\right.$ or $l_{s} \approx j_{s}+\frac{1}{2}$ if $j_{s} \gg 1$ ) and the three $j$ 's around every face satisfy triangle relations (i.e. $\left|j_{1}-j_{2}\right| \leq j_{3} \leq j_{1}+j_{2}$; In this paper, a non-negative half-integer means an element of the set $\left\{0, \frac{1}{2}, 1, \frac{3}{2}, 2, \cdots\right\}$.), then the $6 \mathrm{j}$-symbol $\left\{\begin{array}{lll}j_{1} & j_{2} & j_{3} \\ j_{4} & j_{5} & j_{6}\end{array}\right\}$ is nonzero and approximates the cosine of the Regge action in the large $j$ limit. The positive frequency part of the cosine function is the integrand of the partition function of quantum (Euclidean) general relativity (up to some subtleties concerning the imaginary unit $i \equiv \sqrt{-1}$ ) in the pathintegral formalism. Based on this observation, Ponzano and Regge constructed a "regularized" partition function of quantum gravity on the simplex manifold in terms of $6 j$-symbols.

By constructing the analogous model in terms of a quantum group, Turaev and Viro [2] proved that the value of the partition function of this model is independent of the choice 
of the tetrahedral triangulation of 3-manifold. From this fact it could be said that the partition function of this model is invariant under the renormalization group transformation and depends only on the topology of the manifold. This could be an indication that the model is related to some continuum quantum theory.

By constructing a Hilbert space of state functionals on a 2-space formed and triangulated by the faces of the tetrahedra in the simplex manifold, Ooguri [3] showed that this model is isomorphic to Witten's canonical formulation of $I S O(3)$ Chern-Simons theory [4], which is known to be equivalent to the Ashtekar formulation of general relativity in $(2+1)$-dimensions [5, 6]. (In Ooguri's construction the relevant group is $S O(3)$ rather than $S O(2,1)$.)

These facts suggest that this model may contain some essential information of $(2+1)$ quantum gravity. However, from the model itself, its geometrical meaning is not transparent and this ambiguity makes the model confusing as a physical model. (Another problem of the model is that the partition function has "infrared" divergences if the topology of manifold is $\Sigma \times S^{1}$, where $\Sigma$ is a closed 2-manifold with genus $g>0$ in addition to the (regulated) divergences addressed in this paper. [3]) Therefore, it is desired to find a definition of the model in a geometrically meaningful fashion.

Rovelli [7] pointed out that Ooguri's representation space is closely related to the loop representation [8] of canonical quantum general relativity. [5, 6] Recently, it has been suggested that the partition function of the model may be reconstructed as a sum over surfaces in such a way that its relation to the loop representation of canonical $(2+1)$ quantum (Euclidean) general relativity may be revealed [9].

In this paper, we show that the partition function of the Ponzano-Regge model can be written as a sum over surfaces and suggest a geometrical meaning, in terms of surfaces, for the (regulated) divergences that appear in the partition function.

In fact, what will be done in this paper is the following. We present a partition function which is a sum of products of terms. Each of the terms is a simple combination of variables associated with a vertex, an edge, a face or a tetrahedron of a fixed simplex lattice in a fixed 3-manifold. The values of these variables are determined by simple geometrical relations between the simplex lattice and a family of surfaces. The computations needed in the evaluation of this partition function are very simple combinatorial ones in contrast to the case for the Ponzano-Regge partition function, which includes also combinatrial but quite complicated computations of $6 \mathrm{j}$-symbols in addition to their unclear geometrical meaning. Nevertheless, this partition function is shown to be equivalent to the Ponzano-Regge partition function.

Secondly, the regulator introduced by Ponzano and Regge to regulate divergences appearing in the partition function is reconstructed by means of the variables defined by geometrical relations between the simplex lattice and a family of surfaces and shown to have a geometrical meaning although the regulator was originally constructed algebraically by Ponzano and Regge. We would like to consider this partition function as a geometrically meaningful definition, in terms of surfaces, of the Ponzano-Regge model.

In Sec. 2 we briefly describe the 6j-symbolic formalism of the model due to Ponzano and Regge. In Sec. 3 we present the partition function as a sum over surfaces and show the equivalence to the $6 \mathrm{j}$-symbolic form of the partition function. In Sec. 1 we suggest a geometrical meaning, in terms of surfaces, for the (regulated) divergences that appear in the partition function. We conclude our work in Sec. 5 . 


\section{The Ponzano-Regge model}

The Ponzano-Regge model is defined by the partition function described as follows. Fix a closed 3-manifold $M$. Introduce a tetradedral triangulation $T$ in $M$. Let $S_{0}, S_{1}, S_{2}$ and $S_{3}$ denote the sets of the vertices, edges, faces and tetrahedra in $T$ respectively. Let $N_{0}$, $N_{1}, N_{2}$ and $N_{3}$ denote the numbers of elements of $S_{0}, S_{1}, S_{2}$ and $S_{3}$ respectively. Assign a non-negative half-integer $j_{e}$ to each $e \in S_{1}$. Assign a $6 \mathrm{j}$-symbol to each tetrahedron $t \in S_{3}$ with six non-negative half-integers $j_{s}(s=1,2, \cdots 6)$ already assigned to the edges of $t$ in the way that $\left(j_{1}, j_{2}, j_{3}\right),\left(j_{3}, j_{4}, j_{5}\right),\left(j_{1}, j_{5}, j_{6}\right)$ and $\left(j_{2}, j_{6}, j_{4}\right)$ are the edges of respective faces of $t$. Then the partition function is defined by [1, 2]

$$
Z_{P R}(M, T):=\lim _{N \rightarrow \infty} \sum_{j \leq N} \Lambda^{-N_{0}}(N) \prod_{e \in S_{1}}(-1)^{2 j_{e}}\left(2 j_{e}+1\right) \prod_{t \in S_{3}}(-1)^{-\sum_{s} j_{s}}\left\{\begin{array}{lll}
j_{1} & j_{2} & j_{3} \\
j_{4} & j_{5} & j_{6}
\end{array}\right\}
$$

where sums are taken for all $j$ 's of edges over all the non-negative half-integers less than or equal to $N$. Here $\Lambda(N)$ is introduced to regulate divergences and is defined by

$$
\Lambda(N):=\sum_{k=0, \frac{1}{2}, 1, \cdots N}(2 k+1)^{2},
$$

which behaves as $\Lambda(N) \sim \frac{4}{3} N^{3}$ as $N \rightarrow \infty$. If the $j$ 's of the three edges of any face do not satisfy the triangle relations (i.e. $\left|j_{1}-j_{2}\right| \leq j_{3} \leq j_{1}+j_{2}$ ), then we define the $6 \mathrm{j}$-symbol including these edges to vanish and hence the partition function has many vanishing terms. The variables of this model are the lengths of edges $l=j+\frac{1}{2}$ or $j$ restricted to non-negative half-integers. The way of gluing the tetrahedra, in other words the connectivity and the number of tetrahedra, is fixed in this model.

\section{The partition function in terms of surfaces}

We claim that the partition function of the model for the fixed 3-manifold $M$ with the tetrahedral triangulation $T$ can be written as

$$
\begin{aligned}
Z_{S}(M, T)= & \lim _{N \rightarrow \infty} \sum_{j \leq N} \sum_{K \leq N} \sum_{A, B \leq N}\left(\prod_{v \in S_{0}} \Lambda^{-1}(N)\right)\left(\prod_{e \in S_{1}} \mathcal{L}_{e}\left(j_{e}\right)\right) \times \\
& \left(\prod_{f \in S_{2}} \mathcal{A}_{f}^{-1}\left(K^{f}\right) \prod_{\substack{i, i^{\prime} \\
\left(i<i^{\prime}\right)}} \delta\left[K_{i}^{f}+K_{i^{\prime}}^{f}, j_{e\left(f, i, i^{\prime}\right)}\right]\right) \times \\
& \left(\prod_{t \in S_{3}} \mathcal{V}_{t}\left(A^{t}, B^{t}\right) \prod_{m, n} \delta\left[A_{m}^{t}+B_{n}^{t}, K_{i(t, m, n)}^{f(t, m, n)}\right]\right),
\end{aligned}
$$

with

$$
\mathcal{L}_{e}:=(-1)^{2 j_{e}}\left(2 j_{e}+1\right),
$$




$$
\begin{aligned}
\mathcal{A}_{f} & :=\frac{\left(\sum_{i} 2 K_{i}^{f}+1\right) !}{\prod_{i}\left(2 K_{i}^{f}\right) !} \\
\mathcal{V}_{t} & :=(-1)^{-\sum_{m} A_{m}^{t}+\sum_{n} 2 B_{n}^{t}} \frac{\left(\sum_{m} 2 A_{m}^{t}+\sum_{n} 2 B_{n}^{t}+1\right) !}{\prod_{m}\left(2 A_{m}^{t}\right) ! \prod_{n}\left(2 B_{n}^{t}\right) !}
\end{aligned}
$$

The notation in the equations (4) through (7) is as follows. (Also see Fig. 2.) The $i$ (or $i^{\prime}$ ) is associated with a doublet of edges of a face $f \in S_{2}$ and takes the values 1,2 and 3 . The $m$ is associated with a triplet of edges sharing a single vertex of a tetrahedron $t \in S_{3}$ and takes the values 1, 2, 3 and 4 . The $n$ is associated with a quartet of edges closing a rectangle of a tetrahedron $t \in S_{3}$ and takes the values 1,2 and 3 . The $e\left(f, i, i^{\prime}\right)$ means the edge common to the two doublets $i$ and $i^{\prime}$ of edges of the face $f \in S_{2}$. The $i(t, m, n)$ means the doublet of edges common to the triplet $m$ and the quartet $n$ of edges of the tetrahedron $t \in S_{s}$. The $f(t, m, n)$ means the face on which $i(t, m, n)$ is a doublet of edges. The $j_{e}, K_{i}^{f}, A_{m}^{t}$ and $B_{n}^{t}$ are non-negative half-integer variables, assigned respectively to an edge $e \in S_{1}$, a doublet $i$ of edges of a face $f \in S_{2}$, a triplet $m$ of edges of a tetrahedron $t \in S_{3}$ and a quartet $n$ of edges of a tetrahedron $t \in S_{3}$ and their sums are taken independently for all $e, f, i, t, m$ and $n$ over all the non-negative half-integers less than or equal to $N$. The $\delta$ 's are the Kronecker delta symbols, that is,

$$
\delta[x, y]=\left\{\begin{array}{lll}
1 & \text { if } & x=y \\
0 & \text { if } & x \neq y
\end{array} .\right.
$$

The geometrical meanings of $A_{m}^{t}$ and $B_{n}^{t}$ are as follows. Pick a tetrahedron $t \in S_{3}$. Imagine a surface inside the tetrahedron on which no vertex lies and which divides the tetrahedron into two connected regions. The surface may be closed or open with a boundary on the faces. The surface may intersect some of the edges if it is open. If the surface intersects a triplet of edges sharing a single vertex, then we say the surface is in the class $m$ of type- $A$, where $m$ takes the values $1,2,3$ and 4 corresponding to the triplets of edges defined above. (See Fig. 3.) If the surface intersects a quartet of edges closing a rectangle, then we say the surface is in the class $n$ of type- $B$, where $n$ takes the values 1,2 , and 3 corresponding to the quartets of edges defined above. (See Fig. 3.) Then, given non-negative half-integers $A_{m}^{t}$ and $B_{n}^{t}$, we interpreted them that the tetrahedron $t$ contains $2 A_{m}^{t}$ open surfaces of the class $m$ of type- $A$ and $2 B_{n}^{t}$ open surfaces of the class $n$ of type- $B$. We call the surfaces in these classes the cross-sections of the tetrahedron $t$.

The geometrical meaning of $K_{i}^{f}$ is as follows. Pick a face $f \in S_{2}$. Imagine a curve on the face on which no vertex lies and which divides the face into two connected areas. The curve may be closed or open with two ends at the edges of the face. If the curve has two ends at two different edges, then we say the curve is in the class $i$, where $i$ takes the values 1, 2 and 3 corresponding to the doublets of edges defined above. (See Fig. 3.) Then, given a non-negative half-integer $K_{i}^{f}$, we interpret it that the face $f$ contains $2 K_{i}^{f}$ open curves of the class $i$.

The geometrical meaning of $j_{e}$ is as follows. Pick an edge $e \in S_{1}$. Imagine a point on the edge which does not coincide with any vertex. (See Fig. 3.) Then, given a non-negative half-integer $j_{e}$, we interpret it that the edge $e$ contains $2 j_{e}$ points. (Two points may coincide in position.)

Now, we show that the partition function Eq. (4) can be understood as a sum over (closed) surfaces. First, given a set of non-negative half-integers $\left\{j_{e}, K_{i}^{f}, A_{m}^{t}, B_{n}^{t}\right\}$ for all $e \in S_{1}, i$ on 
$f \in S_{2}$, and $m$ and $n$ in $t \in S_{3}$, place $2 j_{e}$ points on $e \in S_{1}, 2 K_{i}^{f}$ curves of the class $i$ on $f \in S_{2}$ and $2 A_{m}^{t}$ cross-sections of the class $m$ of type- $A$ and $2 B_{n}^{t}$ cross-sections of the class $n$ of type- $B$ in $t \in S_{3}$. By moving the points on edges and/or deforming the curves on faces and the cross-sections in tetrahedra keeping their classes unchanged, we can connect all of them (such that cross-sections are connected by curves and curves are connected by points like in Fig. 3) to form a family of closed surfaces in the manifold $M$ if the set of half-integers satisfies the conditions given by the non-zero Kronecker delta symbols in Eq. (雨). Note that there is no vertex on any of the family of closed surfaces.

Second, suppose a family of closed surfaces on which no vertex lies is given in the manifold $M$. Some of the surfaces may intersect an edge $e \in S_{1}$. Assign to $j_{e}$ half of the number of times the surfaces intersect the edge $e$. Some of the surfaces may intersect a face $f \in S_{2}$ and form curves of the class $i$. Assign to $K_{i}^{f}$ half of the number of curves of the class $i$ formed on the face $f$. Some of the surfaces may intersect a tetrahedron $t \in S_{3}$ and form cross-sections. Assign to $A_{m}^{t}$ half of the number of cross-sections of the class $m$ of type- $A$ formed in the tetrahedron $t$. Assign to $B_{n}^{t}$ half of the number of cross-sections of the class $n$ of type- $B$ formed in the tetrahedron $t$. Therefore, given a family of closed surfaces on which no vertex lies, a set of non-negative half-intergers $\left\{j_{e}, K_{i}^{f}, A_{m}^{t}, B_{n}^{t}\right\}$ is determined.

From the two facts above, we can replace the sums over non-negative half-integers $\left\{j_{e}, K_{i}^{f}, A_{m}^{t}, B_{n}^{t}\right\}$ in Eq. (四) by a sum over families of closed surfaces on which no vertex lies such that every non-negative half-interger in $\left\{j_{e}, K_{i}^{f}, A_{m}^{t}, B_{n}^{t}\right\}$ determined by a family is less than or equal to $N$.

However, some families do not satisfy the conditions given by the non-zero Kronecker delta symbols and hence give rise to vanishing terms in the partition function. We call the closed surfaces of which the families determining non-vanishing terms consist spin-surfaces. This name is based on our interpretation that angular momentum value $j_{e}$ of an edge $e \in S_{1}$ in the $6 \mathrm{j}$-symbolic formulation of the model is determined by the fact that the edge is intersected (or "interacted") $2 j_{e}$ times by the surfaces as if each of them has spin- $\frac{1}{2}$.

Moreover, some families of spin-surfaces may produce an identical set of non-negative half-integers $\left\{j_{e}, K_{i}^{f}, A_{m}^{t}, B_{n}^{t}\right\}$. We define equivalence classes of families of spin-surfaces and say two families of spin-surfaces are equivalent if they produce an identical set of non-negative half-integers $\left\{j_{e}, K_{i}^{f}, A_{m}^{t}, B_{n}^{t}\right\}$. For example, a family of spin-surfaces can be deformed or cut and reconnected in a different way such that its equivalence class is unchanged. (In this sense, intersections of the surfaces do not play any role.) Therefore, the partition function can be expressed as a sum over equivalence classes of families of spin-surfaces (or equivalence classes of spin-surface families in short) such that every non-negative half-integer in $\left\{j_{e}, K_{i}^{f}, A_{m}^{t}, B_{n}^{t}\right\}$ determined is less than or equal to $N$. We can write the partition function formally as

$$
Z_{S}(M, T)=\lim _{N \rightarrow \infty} \sum_{\{\sigma\}_{N}} \prod_{v \in S_{0}} \Lambda^{-1}(N) \prod_{e \in S_{1}} \mathcal{L}_{e} \prod_{f \in S_{2}} \mathcal{A}_{f}^{-1} \prod_{t \in S_{3}} \mathcal{V}_{t}
$$

where $\{\sigma\}_{N}$ stands for the equivalence classes of spin-surface families with a condition that every non-negative half-integers determined from them, $\left\{j_{e}(\sigma), K_{i}^{f}(\sigma), A_{m}^{t}(\sigma), B_{n}^{t}(\sigma)\right\}$, is less than or equal to $N$. Note that this partition function consists of only combinatorial computations.

The next task is to show that our partition function Eq. (四) is in fact equivalent to the 
6j-symbolic form of the partition function Eq. (2). First, insert

$$
\prod_{t \in S_{3}} \prod_{s=1}^{6} \sum_{J_{s}^{t} \leq N} \delta\left[J_{s}^{t}, \sum_{m(s)} A_{m}^{t}+\sum_{n(s)} B_{n}^{t}\right]=1
$$

into Eq. (4), where the sums are taken for all $s$ and $t$ independently over all the non-negative half-integers less than or equal to $N$ and $A_{m}^{t} \leq N$ and $B_{n}^{t} \leq N$. The $s$ is associated with six edges of the tetrahedron $t \in S_{3}$. The $m(s)$ means the two triplets $m$ 's of edges (of the tetrahedron $t$ ) having the edge $s$ and the $n(s)$ means the two quartets $n$ 's of edges (of the tetrahedron $t$ ) having the edge $s$. From the conditions given by the non-zero Kronecker delta symbols in Eq. (10), we can write $A_{m}^{t}+B_{n}^{t}$ in terms of $J_{s}^{t}$ 's, that is,

$$
2\left(A_{m}^{t}+B_{n}^{t}\right)=\left(\sum_{s(t, m, n)} J_{s}^{t}\right)-J_{\bar{s}(t, m, n)}^{t},
$$

where the $s(t, m, n)$ means the two edges in the doublet $i(t, m, n)$ and the $\bar{s}(t, m, n)$ means the edge of $f(t, m, n)$ not in the $i(t, m, n)$. The $s(t, m, n)$ 's and $\bar{s}(t, m, n)$ form a triangle (a face).

Then, Eq. (4) becomes

$$
\begin{aligned}
& Z_{S}(M, T)=\lim _{N \rightarrow \infty} \sum_{j \leq N} \sum_{K \leq N} \sum_{A, B \leq N} \sum_{J \leq N}\left(\prod_{v \in S_{0}} \Lambda^{-1}(N)\right)\left(\prod_{e \in S_{1}} \mathcal{L}_{e}\left(j_{e}\right)\right) \times \\
& \left(\prod_{f \in S_{2}} \mathcal{A}_{f}^{-1}\left(K^{f}\right) \prod_{\substack{i, i^{\prime} \\
\left(i<i^{\prime}\right)}} \delta\left[K_{i}^{f}+K_{i^{\prime}}^{f}, j_{e\left(f, i, i^{\prime}\right)}\right]\right) \times \\
& \left(\prod_{t \in S_{3}} \mathcal{V}_{t}\left(A^{t}, B^{t}\right) \prod_{m, n} \delta\left[\sum_{s(t, m, n)} J_{s}^{t}-J_{\bar{s}(t, m, n)}^{t}, 2 K_{i(t, m, n)}^{f(t, m, n)}\right] \prod_{s=1}^{6} \delta\left[J_{s}^{t}, \sum_{m^{\prime}(s)} A_{m^{\prime}}^{t}+\sum_{n^{\prime}(s)} B_{n^{\prime}}^{t}\right]\right)
\end{aligned}
$$

Notice that the first Kronecker delta symbol in the last parentheses can be rewritten as

$$
\begin{aligned}
& \prod_{t \in S_{3}} \prod_{m, n} \delta\left[\sum_{s(t, m, n)} J_{s}^{t}-J_{\bar{s}(t, m, n)}^{t}, 2 K_{i(t, m, n)}^{f(t, m, n)}\right]=\prod_{f \in S_{2}} \prod_{t(f)} \prod_{i} \delta\left[\sum_{s(f, i)} J_{s}^{t}-J_{\bar{s}(f, i)}^{t}, 2 K_{i}^{f}\right] \\
& =\prod_{f \in S_{2}} \prod_{t(f)} \prod_{\substack{i, i^{\prime} \\
\left(i<i^{\prime}\right)}} \delta\left[J_{s\left(f, i, i^{\prime}\right)}^{t}, K_{i}^{f}+K_{i^{\prime}}^{f}\right]
\end{aligned}
$$

where the $t(f)$ denotes the two adjacent tetrahedra of the face $f$, the $s(f, i)$ means the two edges (of the tetrahedron $t$ ) in the doublet $i$ of the face $f$, the $\bar{s}(f, i)$ means the edge (of the tetrahedron $t$ ) of the face $f$ not in the doublet $i$ of the face $f$, and the $s\left(f, i, i^{\prime}\right)$ means the edge (of the tetrahedron $t$ ) common to the two doublets $i$ and $i^{\prime}$ of edges of the face $f$.

By suming over $K_{i}^{f}$ for all $i$ and $f$, Eq. (12) becomes

$$
\begin{aligned}
& Z_{S}(M, T)=\lim _{N \rightarrow \infty} \sum_{j \leq N} \sum_{J \leq N}\left(\prod_{v \in S_{0}} \Lambda^{-1}(N)\right)\left(\prod_{e \in S_{1}} \mathcal{L}_{e}\left(j_{e}\right)\right) \times \\
& \sum_{A, B \leq N} \prod_{t \in S_{3}}\left(\prod_{f(t)} \mathcal{A}_{f}^{-1 / 2}\left(K^{f}\left(J^{t}\right)\right)\right) \mathcal{V}_{t}\left(A^{t}, B^{t}\right) \prod_{s=1}^{6} \delta\left[J_{s}^{t}, \sum_{m(s)} A_{m}^{t}+\sum_{n(s)} B_{n}^{t}\right] \delta\left[J_{s}^{t}, j_{e(t, s)}\right],
\end{aligned}
$$


where the $f(t)$ denotes the four adjacent faces of the tetrahedron $t$ and the $e(t, s)$ is the edge corresponding to the edge $s$ of the tetrahedron $t$ and $K^{f}\left(J^{t}\right)$ is such that

$$
2 K_{i(t, m, n)}^{f}=\left(\sum_{s(t, m, n)} J_{s}^{t}\right)-J_{\bar{s}(t, m, n)}^{t} .
$$

The sum of the $A_{m}^{t}$ and $B_{n}^{t}$ dependent part for each $t \in S_{3}$ in Eq. (14) is

$$
\begin{aligned}
& \sum_{A^{t}, B^{t} \leq N}\left(\prod_{f(t)} \mathcal{A}_{f}^{-1 / 2}\left(K^{f}\left(J^{t}\right)\right)\right) \mathcal{V}_{t}\left(A^{t}, B^{t}\right) \prod_{s=1}^{6} \delta\left[J_{s}^{t}, \sum_{m(s)} A_{m}^{t}+\sum_{n(s)} B_{n}^{t}\right]
\end{aligned}
$$

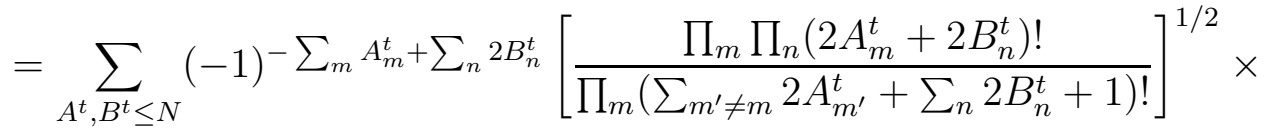

$$
\begin{aligned}
& \frac{\left(\sum_{m} 2 A_{m}^{t}+\sum_{n} 2 B_{n}^{t}+1\right) !}{\prod_{m}\left(2 A_{m}^{t}\right) ! \prod_{n}\left(2 B_{n}^{t}\right) !} \prod_{s=1}^{6} \delta\left[J_{s}^{t}, \sum_{m(s)} A_{m}^{t}+\sum_{n(s)} B_{n}^{t}\right] \\
& =(-1)^{-\sum_{s} J_{s}^{t}}\left\{\begin{array}{lll}
J_{1}^{t} & J_{2}^{t} & J_{3}^{t} \\
J_{4}^{t} & J_{5}^{t} & J_{6}^{t}
\end{array}\right\} .
\end{aligned}
$$

This is a non-zero 6j-symbol. The last step in Eq. (16) may be a surprise, but it agrees with the explicit expression of the $6 \mathrm{j}$-symbol [10], which is

$$
\begin{aligned}
\left\{\begin{array}{ccc}
j_{1} & j_{2} & j_{3} \\
j_{4} & j_{5} & j_{6}
\end{array}\right\}= & (-1)^{j_{1}+j_{2}+j_{4}+j_{5}} \Delta\left(j_{1}, j_{2}, j_{3}\right) \Delta\left(j_{1}, j_{5}, j_{6}\right) \Delta\left(j_{2}, j_{4}, j_{6}\right) \Delta\left(j_{5}, j_{4}, j_{3}\right) \times \\
& \sum_{z}(-1)^{z}\left(j_{1}+j_{2}+j_{4}+j_{5}+1-z\right) !\left[z !\left(j_{3}+j_{6}-j_{1}-j_{4}+z\right) ! \times\right. \\
& \left(j_{3}+j_{6}-j_{2}-j_{5}+z\right) !\left(j_{1}+j_{2}-j_{3}-z\right) !\left(j_{4}+j_{5}-j_{3}-z\right) ! \times \\
& \left.\left(j_{1}+j_{5}-j_{6}-z\right) !\left(j_{2}+j_{4}-j_{6}-z\right) !\right]^{-1},
\end{aligned}
$$

where

$$
\Delta(a, b, c):=\left[\frac{(a+b-c) !(a+c-b) !(b+c-a) !}{(a+b+c+1) !}\right]^{1 / 2}
$$

with $a, b$ and $c$ satisfying the triangle relations, otherwise $\Delta(a, b, c)=0$, and $z$ runs over integer values which do not lead to negative factorials.

Finally, by substituting Eq. (16) into Eq. (14), we find

$$
\begin{aligned}
Z_{S}(M, T)= & \lim _{N \rightarrow \infty} \sum_{j \leq N} \sum_{J \leq N}\left(\prod_{v \in S_{0}} \Lambda^{-1}(N)\right)\left(\prod_{e \in S_{1}} \mathcal{L}_{e}\left(j_{e}\right)\right) \times \\
& \left(\prod_{t \in S_{3}}(-1)^{-\sum_{s} J_{s}^{t}}\left\{\begin{array}{lll}
J_{1}^{t} & J_{2}^{t} & J_{3}^{t} \\
J_{4}^{t} & J_{5}^{t} & J_{6}^{t}
\end{array}\right\} \prod_{s=1}^{6} \delta\left[J_{s}^{t}, j_{e(t, s)}\right)\right) .
\end{aligned}
$$

This is another expression of Eq. (2).

Therefore, our partition function in terms of surfaces is equivalent to the $6 \mathrm{j}$-symbolic partition function of the model. Advantages of the use of the surface formalism may be 
that it may provide a geometrical interpretation of the model in terms of surfaces and that the (regulated) divergences that appear in the partition function may be geometrically understood in terms of surfaces. The first point is deserved for further investigations and, in particular, it is important to clarify what kind of surfaces the spin-surfaces are. The second point is discussed in the next section.

\section{Divergences}

In the $6 \mathrm{j}$-symbolic partition function Eq. (2), $\Lambda(N)$ is present to regulate divergences. In the surface formalism described in the previous section, the same regulator $\Lambda(N)$ is used although its meaning has not yet been explained. In this section we analyze the regulator in terms surfaces and suggest a geometrical meaning for the divergences regulated.

Ponzano and Regge introduced the regulator $\Lambda(N)$ defined by Eq. (3) in the following way. [1] Pick a tetrahedron from the tetrahedral triangulation $T$. By placing one more vertex and four edges in this tetrahedron, make a finer triangulation consisting of four tetrahedra in the original tetrahedron and the other tetrahedra in T. (See Fig. \&.) Assign a non-negative half-integer $j_{s}$ or $j_{a}$ to each of the ten edges of the four tetrahedra. The $s=1,2, \cdots, 6$ denote the edges on the boundary, consisting of the four faces of the original tetrahedron, and the $a=7,8,9,10$ denote the interior edges added. In addition to the four faces of the original tetrahedron, there are six more faces formed by $\left(j_{1}, j_{9}, j_{8}\right),\left(j_{2}, j_{7}, j_{9}\right),\left(j_{3}, j_{8}, j_{7}\right),\left(j_{4}, j_{7}, j_{10}\right)$, $\left(j_{5}, j_{8}, j_{10}\right)$, and $\left(j_{6}, j_{10}, j_{9}\right)$. Assign a $6 \mathrm{j}$-symbol to each of the four tetrahedra. Then the $6 \mathrm{j}$-symbols satisfy the relation

$$
\begin{aligned}
& \sum_{j_{7}, j_{8}, j_{9}, j_{10}}\left(\prod_{a=7}^{10}(-1)^{2 j_{a}}\left(2 j_{a}+1\right)\right)(-1)^{-\sum_{s=1}^{6} 2 j_{s}-\sum_{a=7}^{10} 3 j_{a} \times} \\
& \left\{\begin{array}{lll}
j_{1} & j_{2} & j_{3} \\
j_{7} & j_{8} & j_{9}
\end{array}\right\}\left\{\begin{array}{lll}
j_{6} & j_{4} & j_{2} \\
j_{7} & j_{9} & j_{10}
\end{array}\right\}\left\{\begin{array}{lll}
j_{5} & j_{3} & j_{4} \\
j_{7} & j_{10} & j_{8}
\end{array}\right\}\left\{\begin{array}{lll}
j_{1} & j_{5} & j_{6} \\
j_{10} & j_{9} & j_{8}
\end{array}\right\} \\
= & (-1)^{-\sum_{s=1}^{6} j_{s}}\left\{\begin{array}{lll}
j_{1} & j_{2} & j_{3} \\
j_{4} & j_{5} & j_{6}
\end{array}\right\}\left(\lim _{N \rightarrow \infty} \Lambda(N)\right),
\end{aligned}
$$

where the sums are taken over all the non-negative half-integers. Because of this relation, the partition function is made invariant under the change of tetrahedral triangulations. The $\Lambda(N)$ is divergent as $N \rightarrow \infty$ and the presence of $\Lambda^{-1}(N)$ for the vertex added in the partition function divides the divergence away.

However, from Eq. (20) of the 6j-symbolic formalism, the geometrical meaning of $\Lambda(N)$ is unclear since no variable like $k$ in Eq. (3) is assigned to any vertex in the model. In order to find a geometrical meaning of $\Lambda(N)$ in the surface formalism, consider the case that $j_{s}(s=1,2, \cdots 6)$ on the boundary vanish (note $\left\{\begin{array}{lll}0 & 0 & 0 \\ 0 & 0 & 0\end{array}\right\}=1$ ) and rewrite the $6 \mathrm{j}$-symbols in terms of surfaces using Eq. (16). Then the Eq. (20) becomes

$$
\begin{aligned}
& \lim _{N \rightarrow \infty} \Lambda(N) \\
& =\sum_{j_{7}, j_{8}, j_{9}, j_{10}}\left(\prod_{a=7}^{10}(-1)^{2 j_{a}}\left(2 j_{a}+1\right)\right)(-1)^{-\sum_{a=7}^{10} 3 j_{a}} \times
\end{aligned}
$$




$$
\begin{aligned}
& \left\{\begin{array}{ccc}
0 & 0 & 0 \\
j_{7} & j_{8} & j_{9}
\end{array}\right\}\left\{\begin{array}{ccc}
0 & 0 & 0 \\
j_{7} & j_{9} & j_{10}
\end{array}\right\}\left\{\begin{array}{ccc}
0 & 0 & 0 \\
j_{7} & j_{10} & j_{8}
\end{array}\right\}\left\{\begin{array}{ccc}
0 & 0 & 0 \\
j_{10} & j_{9} & j_{8}
\end{array}\right\} \\
& =\lim _{N \rightarrow \infty} \sum_{k \leq N}\left(\prod_{e}(-1)^{2 k}(2 k+1)\right)\left(\prod_{f}(2 k+1)^{-1}\right)\left(\prod_{t}(-1)^{-k}(2 k+1)\right),
\end{aligned}
$$

where $k$ is a non-negative half-integer variable determined by equivalence classes of spinsurface families with spin-surfaces enclosing the interior vertex, intersecting the four interior edges and forming a cross-section of type- $A$ in each of the four interior tetrahedra (See Fig. 1; there are only equivalence classes of spin-surface families consisting of these spinsurfaces when $j_{s}$ 's on the boundary are zero.) and $e, f$ and $t$ stand for the interior edges, the interior faces and the interior tetrahedra respectively.

By letting $n_{0}, n_{1}$ and $n_{2}$ denote the numbers of the interior edges, the interior faces and the interior tetrahedra respectively and using the relation $2 n_{1}=3 n_{2}$, Eq. (21) can be rewritten as

$$
\begin{aligned}
\Lambda(N) & =\sum_{k \leq N}\left[(-1)^{2 k}(2 k+1)\right]^{n_{0}}(2 k+1)^{-n_{1}}\left[(-1)^{-k}(2 k+1)\right]^{n_{2}} \\
& =\sum_{k \leq N}(-1)^{2 k\left(n_{0}-n_{1}+n_{2}\right)}(2 k+1)^{n_{0}-n_{1}+n_{2}} \\
& =\sum_{k \leq N}\left[(-1)^{2 k}(2 k+1)\right]^{\chi}
\end{aligned}
$$

where $\chi:=n_{0}-n_{1}+n_{2}=2(1-g)$ is the Euler characteristic of a closed surface with the genus $g$ embedded in a 3-space. In our case here $n_{0}=4, n_{1}=6, n_{2}=4$ and hence $g=0$ and $\chi=2$. Therefore, the $\Lambda(N)$ defined in Eq. (3) has been recovered here with geometrical meanings: (i) The $k$ is the variable determined by equivalence classes of families

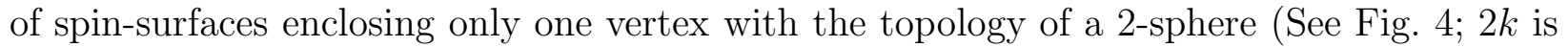
the number of such spin-surfaces), and (ii) the exponent in the right hand side of Eq. (3) is the Euler characteristic of each of these spin-surfaces.

Note that Eq.(22) is divergent as $N \rightarrow \infty$ if $\chi=2$ or 0 . In other words, surfaces with $\chi=2$ and 0 may be origins of divergences. This conjecture may give a hint to clarifying the spin-surfaces.

\section{Conclusions}

In this paper, we showed that the partition function of the Ponzano-Regge quantum gravity model can be written as a sum over surfaces in a $(2+1)$ dimensional space-time. We called the surfaces playing roles in the partition function spin-surfaces because they were interpreted to determine the angular momentum values of edges in the $6 \mathrm{j}$-symbolic form of the partition function by intersecting (or "interacting") the edges as if each surface has spin$\frac{1}{2}$. We analyzed the regulator introduced by Ponzano and Regge by means of the surface formalism and suggested a geometrical meaning, in terms of surfaces, for the (regulated) divergences that appear in the partition function.

We may think that the existences of this surface formulation of the model and this way of understanding the (regulated) divergences that appear in the partition function may provide 
a further interest to the model. It may be hoped that this surface formulation may provide a geometrical way of investigating the otherwise very subtle model and hence another way of studying well explored problems in $(2+1)$ quantum gravity from a slightly different angle.

\section{Acknowledgements}

The author has benefited from discussions with Carlo Rovelli. The author also thanks Lee Smolin for helpful suggestions.

\section{References}

[1] G. Ponzano, T. Regge, in Spectroscopy and Group Theoretical Methods in Physics, ed. by F. Block, North-Holland, Amsterdam (1968).

[2] V. Turaev, O. Viro, Topology 31, 865 (1992).

[3] H. Ooguri, Nucl. Phys. B382, 276 (1992).

[4] E. Witten, Nucl. Phys. B311, 46 (1988).

[5] A. Ashtekar, V. Husain, C. Rovelli, J. Samuel, L. Smolin, Class. Quantum Grav. 6, L185 (1989).

[6] A. Ashtekar, Lectures on non-perturbative canonical gravity, World scientific, Singapore (1991).

[7] C. Rovelli, Phys. Rev. D 48, 2702 (1993).

[8] C. Rovelli, L. Smolin, Phys. Rev. Lett. 61, 1155 (1988); Nucl. Phys. B331, 80 (1990).

[9] J. Iwasaki, A reformulation of the Ponzano-Regge quantum gravity model in terms of surfaces, University of Pittsburgh preprint, gr-qc/9410010 (January, 1994).

[10] D.M. Brink, G.R. Satchler, Angular Momentum, Oxford University Press (1962). 


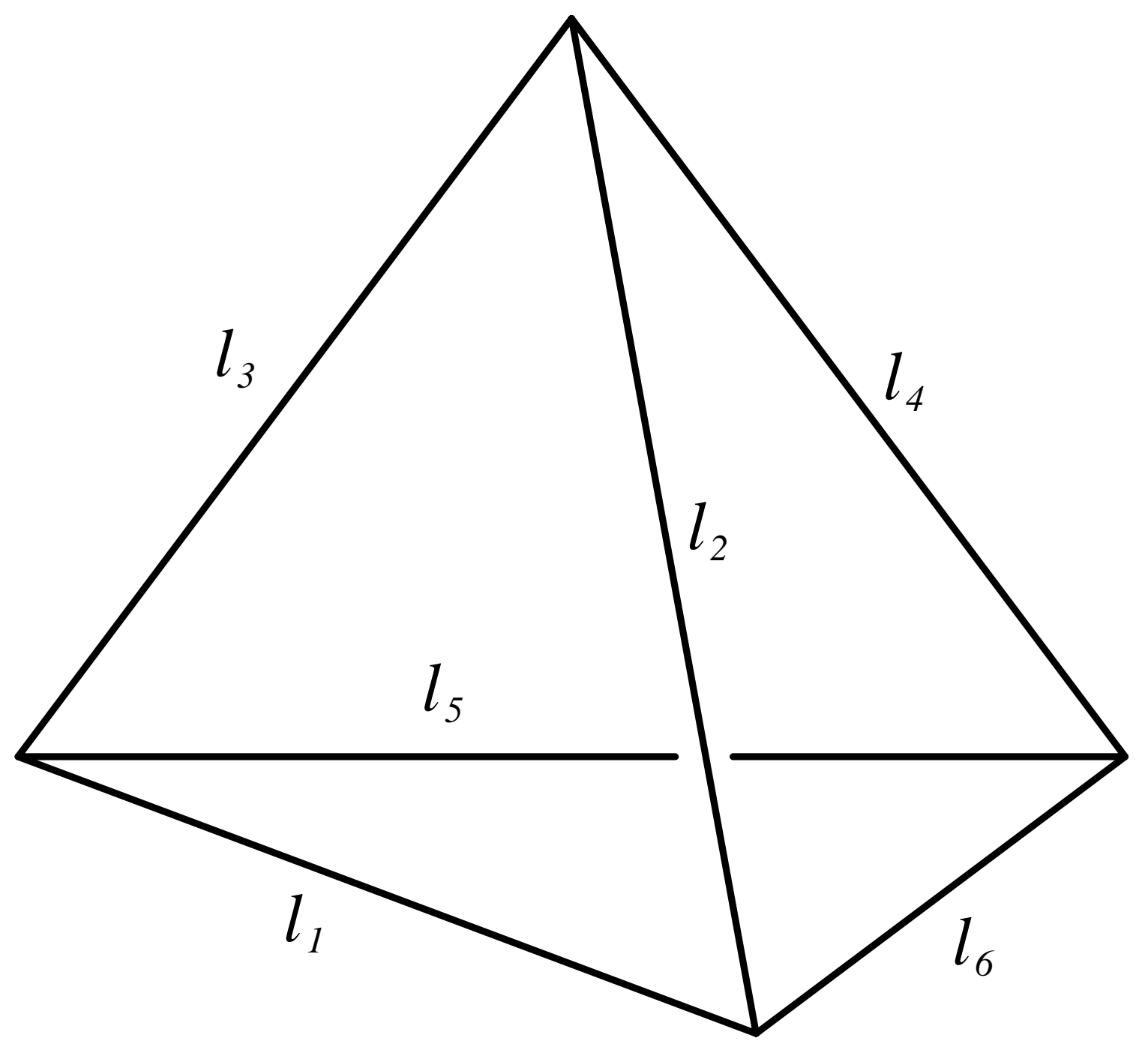

Figure 1: The tetrahedron with edge lengths $l_{i}(i=1,2, \cdots, 6)$. 
Face $f$ :

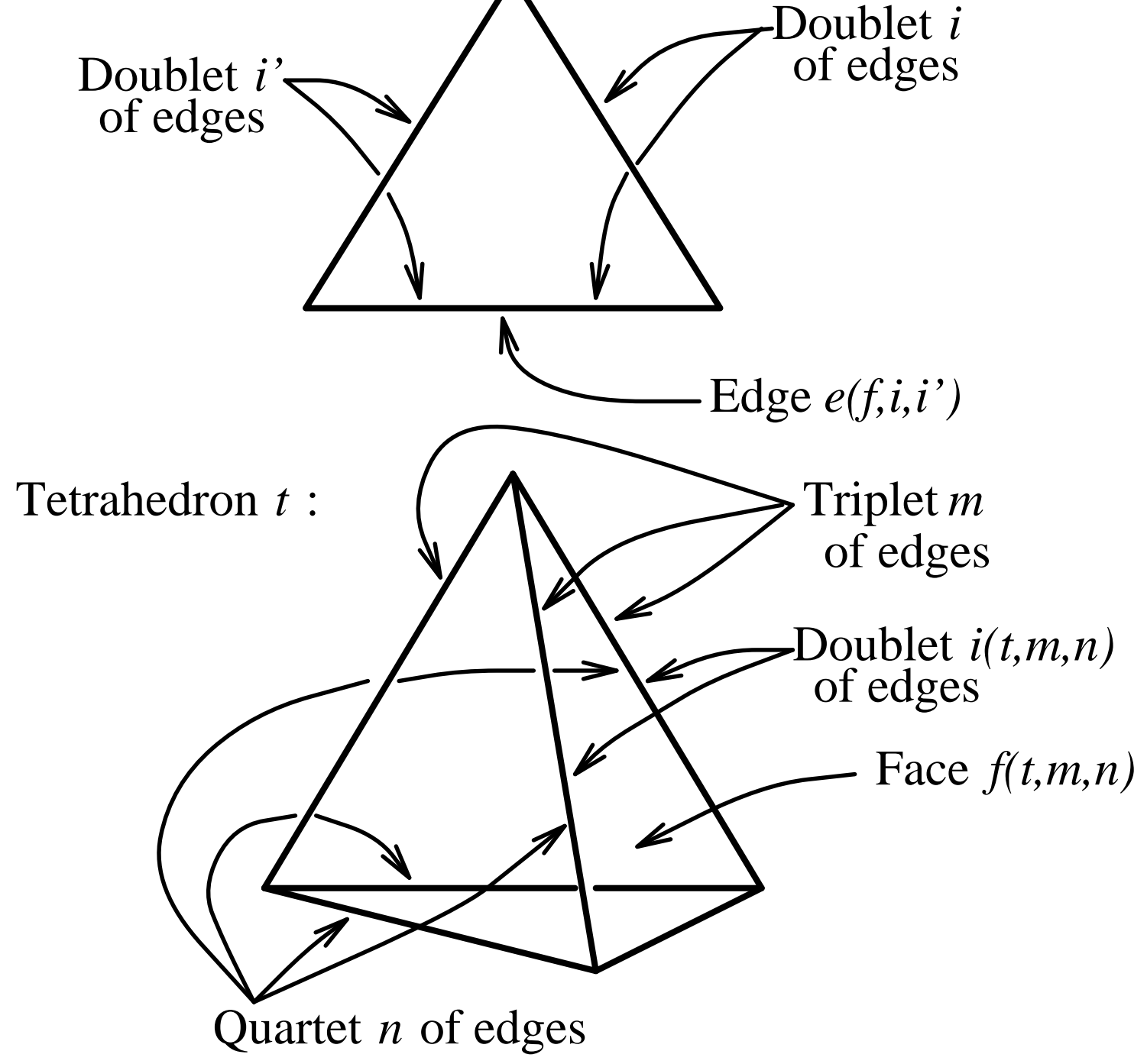

Figure 2: Nomenclature for the partition function. 

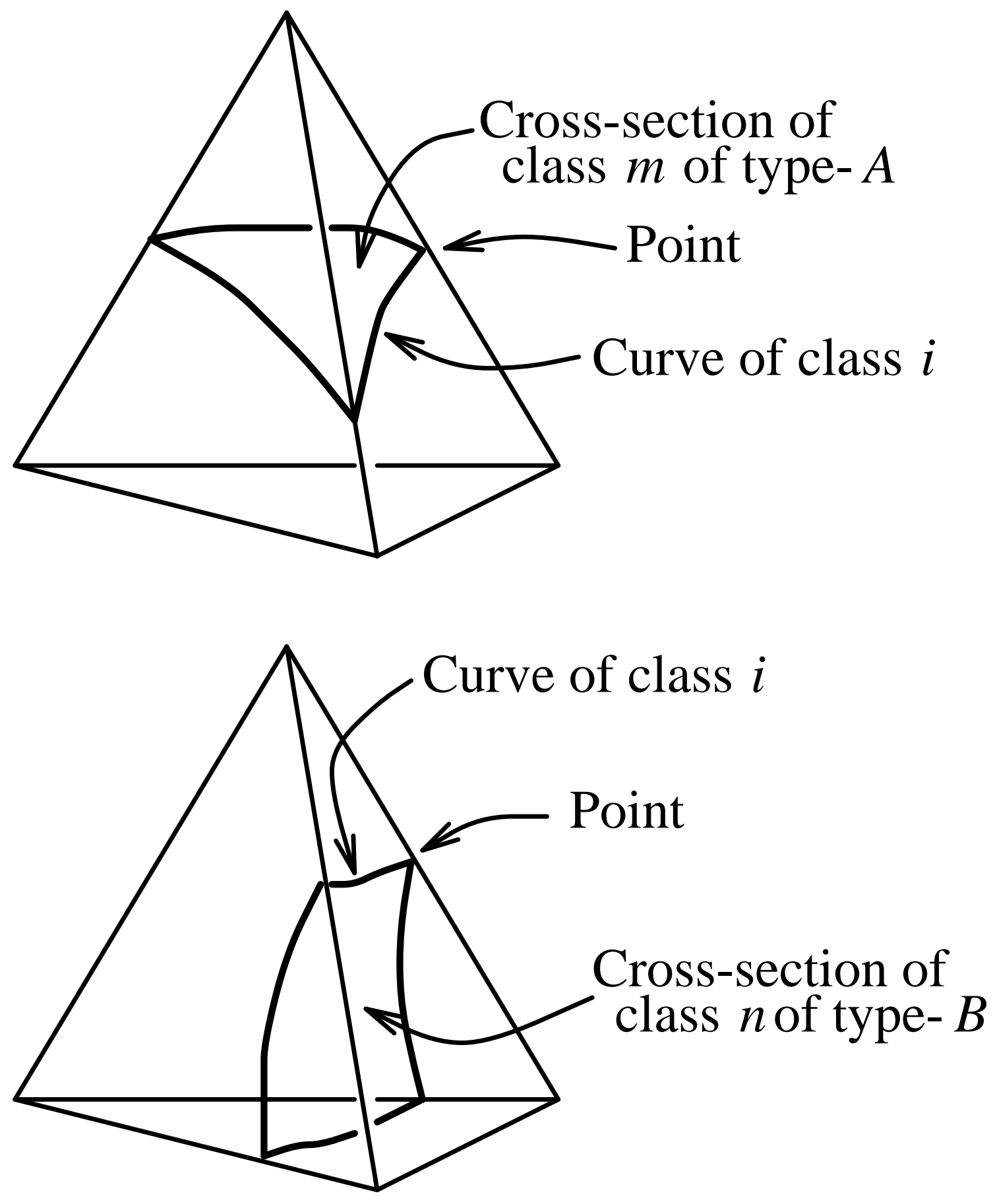

Figure 3: Points on edges, curves on faces and cross-sections in tetrahedra. 


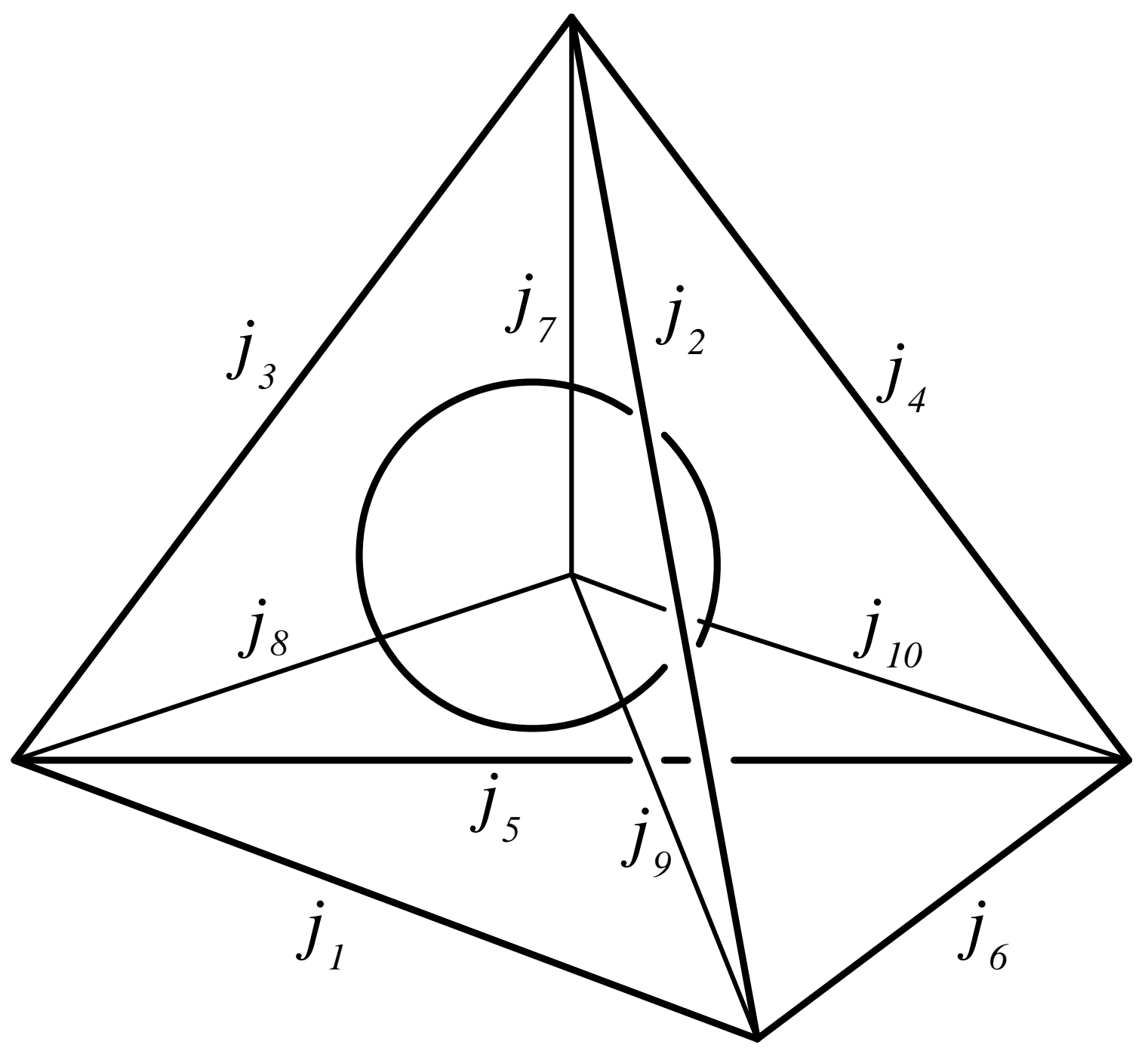

Figure 4: The decomposition of a tetrahedron to four smaller tetrahedra, and a spin-surface contributing to $\Lambda(N)$. 